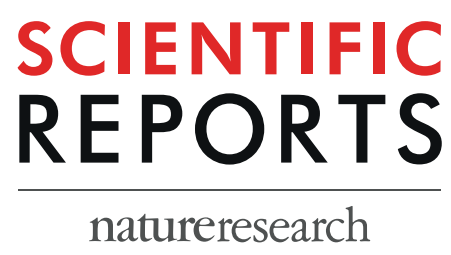

\title{
OPEN Analysis of Patients with Urolithiasis Visiting the Emergency Department between 2014 and 2016 in Korea: Data from the National Emergency Department Information System
}

\author{
Jong Wook Kim $\mathbb{1}^{1}$, Jung-Youn $\mathrm{Kim}^{2}$, Sun Tae $\mathrm{Ahn}^{1}$, Mi Mi Oh${ }^{1}$, Du Geon Moon $\mathbb{D}^{1}$ \& \\ Hong Seok Park $\mathbb{1}^{1^{*}}$
}

This study investigated the characteristics of patients with urolithiasis visiting an emergency department based on a national database system in Korea. This study spanned a period of three years from January 1, 2014 to December 31, 2016. A retrospective census was conducted using the National Emergency Department Information System for urolithiasis patients. Patient data, including age, sex, insurance type, emergency department visit date and time, discharge date and time, emergency department treatment result, visit flow, and hospitalization route, were extracted and analyzed. Overall, 103,981, 112,083, and 120,647 patients/year during the 2014-2016 study period visited an emergency department with a diagnosis related to urolithiasis. Total monthly emergency department visits ranged from 35,927 in August (highest) to 24,008 in February. Overall, 13.2\% of patients were hospitalized and the hospitalization rate was stable (estimated annual percent change) over the study period. Patients aged $<9$ years or $\geq 70$ years and those with medical aid had higher hospitalization rates. A higher number of visits occurred in the hot season, on weekends, and in the 6 a.m. and 8 p.m. time slots. This nationwide study revealed that the percentage of patients visiting an emergency department with urolithiasis was higher in August, in the early morning, and at weekends.

Patients who visit emergency department (ED) due to acute abdominal pain are often diagnosed with urolithiasis. The chief complaint of patients with urolithiasis is a sudden onset of flank pain, lower back pain to the genitalia, and hematuria ${ }^{1}$. Urolithiasis is a common disease worldwide. The prevalence of urolithiasis in Korea has been reported to be approximately $5.7 \%^{2}$. According to literature, the life-time development risk is $12 \%$ (in men) and $6 \%$ (in women), and the lifetime cumulative incidence ranges from $5-10 \%{ }^{1,3,4}$. The 10 -year recurrence is high, at approximately $42-50 \%$.5.6. Most urolithiasis patients receive acute treatment in $\mathrm{ED}^{7,8}$.

The causes of urolithiasis are multifactorial. Numerous epidemiological studies indicate sex, race, age, climate, occupation, and obesity influence occurrence ${ }^{9,10}$. Except for urolithiasis patients who visit a hospital for follow-up observation, most patients receive treatment in $\mathrm{ED}$ for pain control ${ }^{7}$. Therefore, $\mathrm{ED}$ is a very important contact point for treatment of urolithiasis patients. Although the overall prevalence of urolithiasis has been examined previously, few studies have evaluated the characteristics of urolithiasis patients visiting ED. As most urolithiasis patients visit $\mathrm{ED}$, their use of $\mathrm{ED}$ should be analyzed to improve treatment and management at limited $\mathrm{ED}$ facilities.

In the present study, information was extracted relative to urolithiasis patients from a national database of ED. The data were surveyed and analyzed for epidemiologic characteristics of urolithiasis patients visiting ED over a three-year period. Based on the general characteristics analyzed, the state of use of ED and hospital resources was 
examined. This study attempted to provide essential data useful for patient treatment and efficient management of limited resources.

\section{Materials and Methods}

Study design and database. This study utilized National Emergency Department Information System (NEDIS) for secondary data analysis. NEDIS is an emergency information network operated by the government (Ministry of Health \& Welfare) since 2003 and is controlled by National Emergency Medical Center. NEDIS includes clinical and administrative data of all patients who have visited ED across the country ${ }^{11}$. In Korea, a national health insurance service is provided that covers approximately $98 \%$ of the total population. Thus, national data are considered influential. Emergency centers across the nation undergo approval assessment yearly to be approved as an emergency service institution. Essentially, they are required to digitalize all data items of NEDIS and transmit them for assessment. Thus, it may be assumed that the data used in this study reflect the data of all ED in Korea.

Data collection. A retrospective census of urolithiasis patients who visited ED over the three-year study period (January 1, 2014 to December 31, 2016) was conducted. Patient data, including age, sex, insurance type, ED visit date and time, discharge date and time, ED treatment outcomes, visit route, and hospitalization path, were extracted from the national database and were analyzed after an official application for access to data. The corresponding ICD codes used for diagnoses are N20, N21, N22 and N23.

Outcome measures. General characteristics of the patients were compared. Hospitalized patients and discharged patients were examined to analyze their epidemiologic characteristics, such as visit date and time, age, sex, treatment outcome, the number of individual visits by the same patient compared to all ED visits, the length of stay in the ED, hospitalization rate, and main visit duration (hours and months).

Statistical analyses. For all variables, the hospitalization group was compared to the non-hospitalization group. All statistical analyses were performed using SPSS software (version 20.0; IBM SPSS, Armonk, NY, USA) and Excel (Microsoft Corporation, Redmond, WA, USA). For frequency analysis, a one-way analysis of variance test was conducted. Null hypotheses of no difference were rejected with p-values $<0.05$. Data are expressed as $n$ $(\%)$, mean \pm standard deviation (SD).

Ethics statement. This research received approval from the institutional review board of Korea University Guro Hospital (No. 2018GR0136). The requirement for informed consent from the participants was waived by the board.

\section{Results}

The number of urolithiasis patients visiting the EDs from 2014-2016 was 336,711. Of these, patients who required hospitalization for treatment accounted for $13.2 \%$, while $86.8 \%$ were discharged after treatment. The average age of the patients overall was $47.8 \pm 15$ years, and hospitalized patients were older on average than those discharged (53.9 \pm 17.2 vs. $46.8 \pm 14.4$ years). Men outnumbered women $(222,659$ [66.1\%] vs. 114,052 [33.9\%]) and $11 \%$ of men required hospitalization, whereas only $17.3 \%$ of women were hospitalized. The hospitalization rate did not differ according to the grade of the emergency center.

Although patients visiting EDs on weekends numbered 110,431 (32.8\%), the hospitalization rate was higher on weekdays ( $13.7 \%$ vs. $12.1 \%$ ). Patients with commercial insurance coverage or a specific insurance type, such as car insurance, had higher hospitalization rates, and patients with Medicaid insurance had a higher hospitalization rate than those who had general Medicare. The number of patients who visited EDs as individual walk-in patients was higher than those transferred from other hospitals or by ambulance. In cases of hospitalization, patients arriving by ambulance outnumbered those arriving by other means. Patients who were hospitalized remained in the ED longer and had a longer time from occurrence to a visit. The number of urolithiasis patients increased steadily per year of the study (103,981 vs. 112,083 vs. 120,647$)$, while the number of patients who visited the nation's EDs overall also increased constantly per year (8,033,594 vs. 8,512,834 vs. 9,268,112). Urolithiasis patients accounted for $1.3 \%$ of those visiting the EDs (Table 1 ).

A higher number of urolithiasis patients visited the EDs between 6-7 a.m. and 8-10 p.m. Most patients who visited the EDs during daytime were hospitalized (Fig. 1).

The largest number of patients visited the EDs in August $(35,927)$, followed by September $(32,099)$, July $(30,695)$, and May $(30,624)$. In terms of seasons, the highest visits occurred in summer. February had the fewest visits (24,008) (Fig. 2). The largest number of patient visits occurred on Saturdays and Sundays (Fig. 3).

Compared to other age groups, patients in their 50s comprised the largest number of patients visiting the EDs, followed by patients in their 40s and 30s (Table 1 ), while patients $<9$ years $(30.4 \%$ ) and those in their 70 s and 80 s and older had a remarkably high hospitalization rate (Fig. 4).

The stay in the ED was $2.6 \pm 5.11$ hours on average and for hospitalized patients this stay was longer $(5.3 \pm 28.1$ vs. $2.2 \pm 5.1$ ).

The annual rate of hospitalized patients was not significantly different ( $12.8 \%$ vs. $13.3 \%$ vs. $13.3 \%$ from 2014 to 2016, respectively). The number of urolithiasis patients visiting the EDs increased steadily annually from 2014 to 2016 (103,981 vs. 112,083 vs. 120,647); However the proportion of urolithiasis patients among all ED patients remained constant at $1.3 \%$ for all three years. 


\begin{tabular}{|c|c|c|c|c|c|c|}
\hline & Total & Admission ( & & Treated and & & P value \\
\hline Patients, n (\%) & 336,711 & 44,323 & 13.2 & 292,388 & 86.8 & \\
\hline Age $($ mean \pm SD) & $47.8 \pm 15.0$ & $53.9 \pm 17.2$ & & $46.8 \pm 14.4$ & & $<0.001$ \\
\hline Sex n (\%): & & & & & & $<0.001$ \\
\hline Male & 222,659 & 24,576 & 11.0 & 198,083 & 89.0 & \\
\hline Female & 114,052 & 19,747 & 17.3 & 94,305 & 82.7 & \\
\hline No. ED* type (\%): & & & & & & $<0.001$ \\
\hline Regional emergency medical center & 47,096 & 5,992 & 12.7 & 41,104 & 87.3 & \\
\hline Sub-specialty emergency medical center & 700 & 114 & 16.3 & 586 & 83.7 & \\
\hline Local emergency medical center & 106,756 & 14,535 & 13.6 & 92,221 & 86.4 & \\
\hline Local emergency medical agency & 181,063 & 23,463 & 13.0 & 157,600 & 87.0 & \\
\hline Unknown & 1,096 & 219 & 20.0 & 877 & 80.0 & \\
\hline Most common diagnosis code: & & & & & & $<0.001$ \\
\hline N201 (Calculus of ureter) & 262,489 & 27,104 & 10.3 & 235,385 & 89.7 & \\
\hline N219 (Calculus of lower urinary tract, unspecified) & 17,368 & 1,442 & 8.3 & 15,926 & 91.7 & \\
\hline Patient status: & & & & & & $<0.001$ \\
\hline Urgent & 257,202 & 36,586 & 14.2 & 220,616 & 85.8 & \\
\hline Non-urgent & 79,480 & 7,737 & 9.7 & 71,743 & 90.3 & \\
\hline Others & 29 & 0 & 0.0 & 29 & 100.0 & \\
\hline Weekend ED visit, $\mathrm{n}(\%)$ : & & & & & & $<0.001$ \\
\hline No & 226,280 & 31,012 & 13.7 & 195,268 & 86.3 & \\
\hline Yes & 110,431 & 13,311 & 12.1 & 97,120 & 87.9 & \\
\hline Type of insurance, n (\%) & & & & & & $<0.001$ \\
\hline Medicare & 320,692 & 41,226 & 12.9 & 279,466 & 87.1 & \\
\hline Medicaid 1 & 7,044 & 2,073 & 29.4 & 4,971 & 70.6 & \\
\hline Medicaid 2 & 2,437 & 327 & 13.4 & 2,110 & 86.6 & \\
\hline Other & 1,465 & 170 & 11.6 & 1,295 & 88.4 & \\
\hline Unknown & 214 & 19 & 8.9 & 195 & 91.1 & \\
\hline Private & 2 & 0 & 0.0 & 2 & 100.0 & \\
\hline Commercial & 101 & 54 & 53.5 & 47 & 46.5 & \\
\hline Uninsured & 4,456 & 282 & 6.3 & 4,174 & 93.7 & \\
\hline Car & 300 & 172 & 57.3 & 128 & 42.7 & \\
\hline Route of visit & & & & & & $<0.001$ \\
\hline Direct visit & 315,792 & 36,363 & 11.5 & 279,429 & 88.5 & \\
\hline Transferred-in & 17,577 & 6,603 & 37.6 & 10,974 & 62.4 & \\
\hline From outpatient area & 2,820 & 1,319 & 46.8 & 1,501 & 53.2 & \\
\hline Others & 522 & 38 & 7.3 & 484 & 92.7 & \\
\hline Mode of arrival, $\mathrm{n}(\%)$ & & & & & & $<0.001$ \\
\hline Private transportation (car) & 260,304 & 30,419 & 11.7 & 229,885 & 88.3 & \\
\hline Public ambulance service & 50,010 & 7,720 & 15.4 & 42,290 & 84.6 & \\
\hline Walk-in & 18,592 & 2,553 & 13.7 & 16,039 & 86.3 & \\
\hline Private ambulance service & 3,277 & 2,065 & 63.0 & 1,212 & 37 & \\
\hline Other hospital ambulances & 1,640 & 1,103 & 67.3 & 537 & 32.7 & \\
\hline Aeromedical transport & 178 & 23 & 12.9 & 155 & 87.1 & \\
\hline Public transportation (e.g., police car) & 167 & 15 & 9.0 & 152 & 91 & \\
\hline Other & 2,543 & 425 & 16.7 & 2,118 & 83.3 & \\
\hline Length of ED stay & $2.6 \pm 5.11$ & $5.3 \pm 28.1$ & & $2.2 \pm 5.1$ & & $<0.001$ \\
\hline Time from onset to ED arrival & $18.1 \pm 160.4$ & $39.6 \pm 160.4$ & & $14.9 \pm 160.4$ & & $<0.001$ \\
\hline Year, n (\%) & & & & & & $<0.001$ \\
\hline 2014 & $103,981(1.3 \%)$ & 13,320 & 12.8 & 90,661 & 87.2 & \\
\hline 2015 & $112,083(1.3 \%)$ & 14,905 & 13.3 & 97,178 & 86.7 & \\
\hline 2016 & $120,647(1.3 \%)$ & 16,098 & 13.3 & 104,549 & 86.7 & \\
\hline
\end{tabular}

Table 1. Characteristics of patients diagnosed with urolithiasis in the emergency department according to NEDIS data from 2014 to 2016. NEDIS, National Emergency Department Information System. 
25000

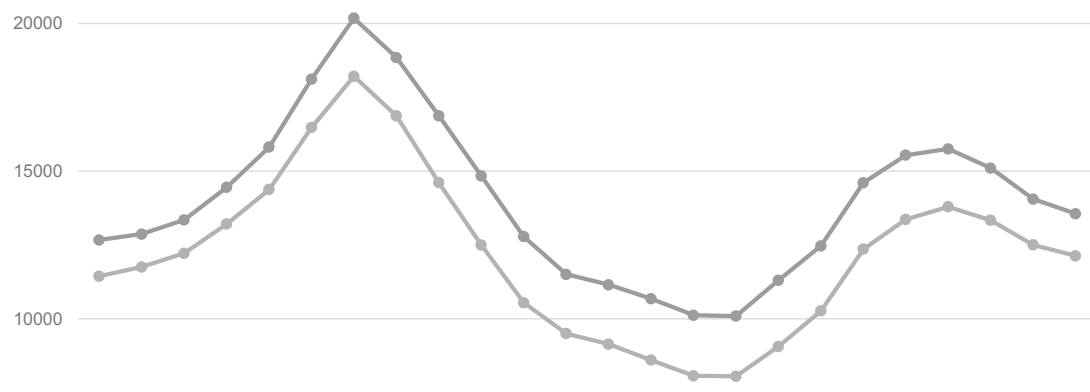

5000

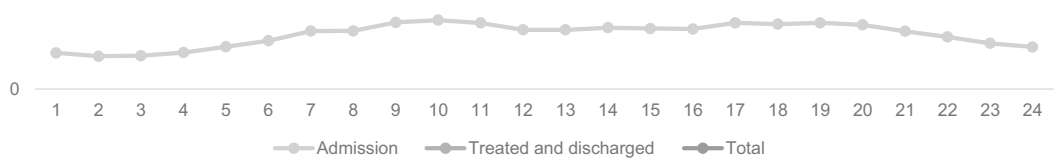

Figure 1. Number of Emergency Department visits by time of day. Total number of ED visits, admissions, treated and discharged patients for urolithiasis in Korea from 2014 to 2016 in the NEDIS stratified by time of day in hours.

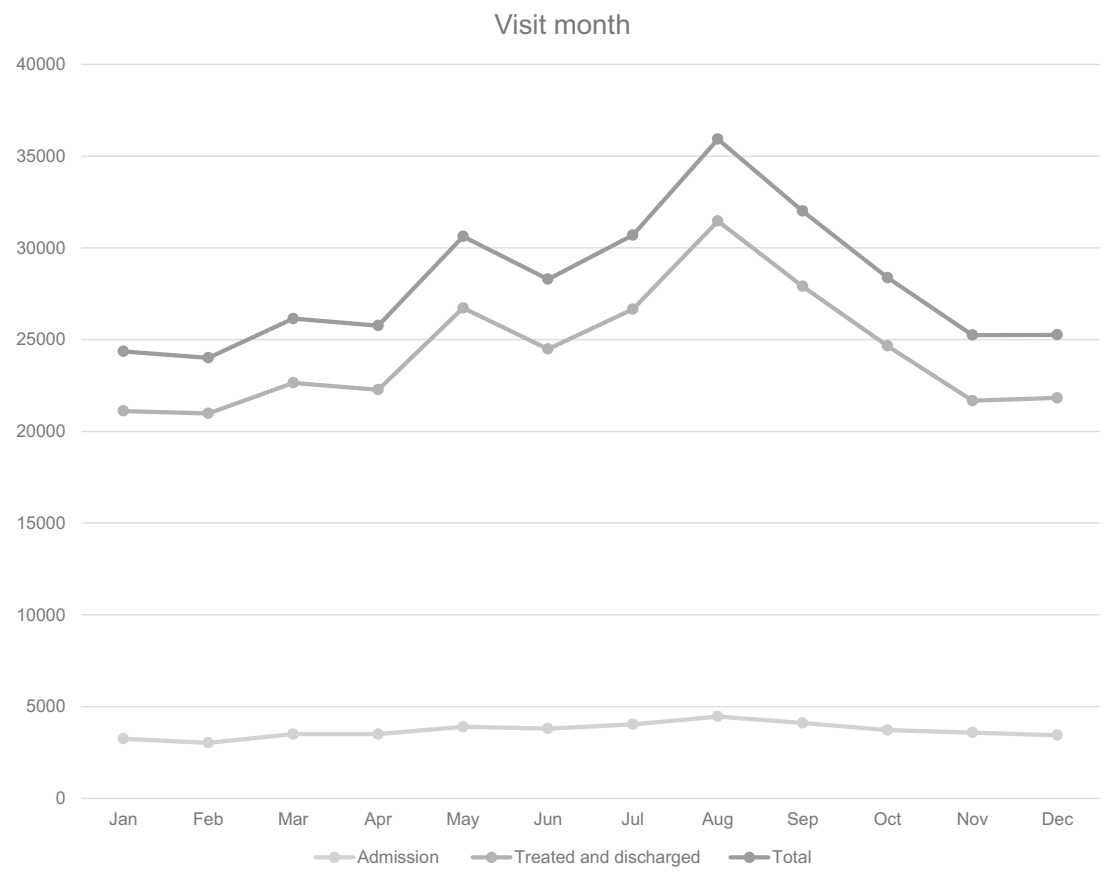

Figure 2. Number of Emergency Department visits by month. Total number of Emergency Department visits, admissions, treated and discharged patients for urolithiasis in Korea from 2014 to 2016 in NEDIS stratified by month.

\section{Discussion}

This study is meaningful because it examined the epidemiologic characteristics of urolithiasis patients visiting ED and hospitalized patients in Korea based on a national database. Severe acute flank pain is the most common symptom of urolithiasis. Thus, if urolithiasis occurs, patients are highly likely to visit ED. However, there are few studies about nation-wide information on such visits to ED. 


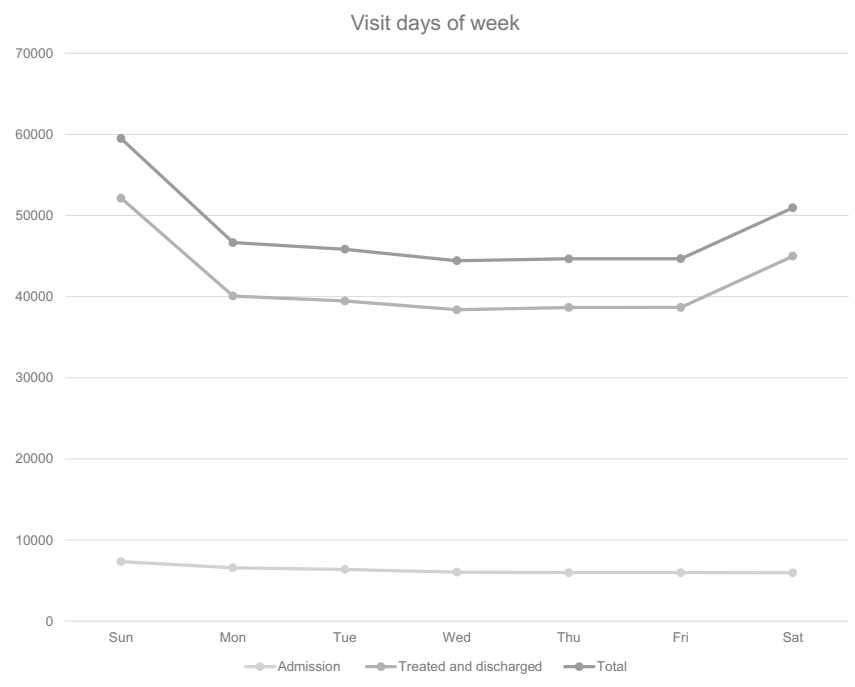

Figure 3. Number of Emergency Department visits subdivided by the day of the week. Total number of Emergency Department visit, admission, treated, and discharged patient for urolithiasis in Korea in 2014 to 2016 in NEDIS stratified by day of the week.

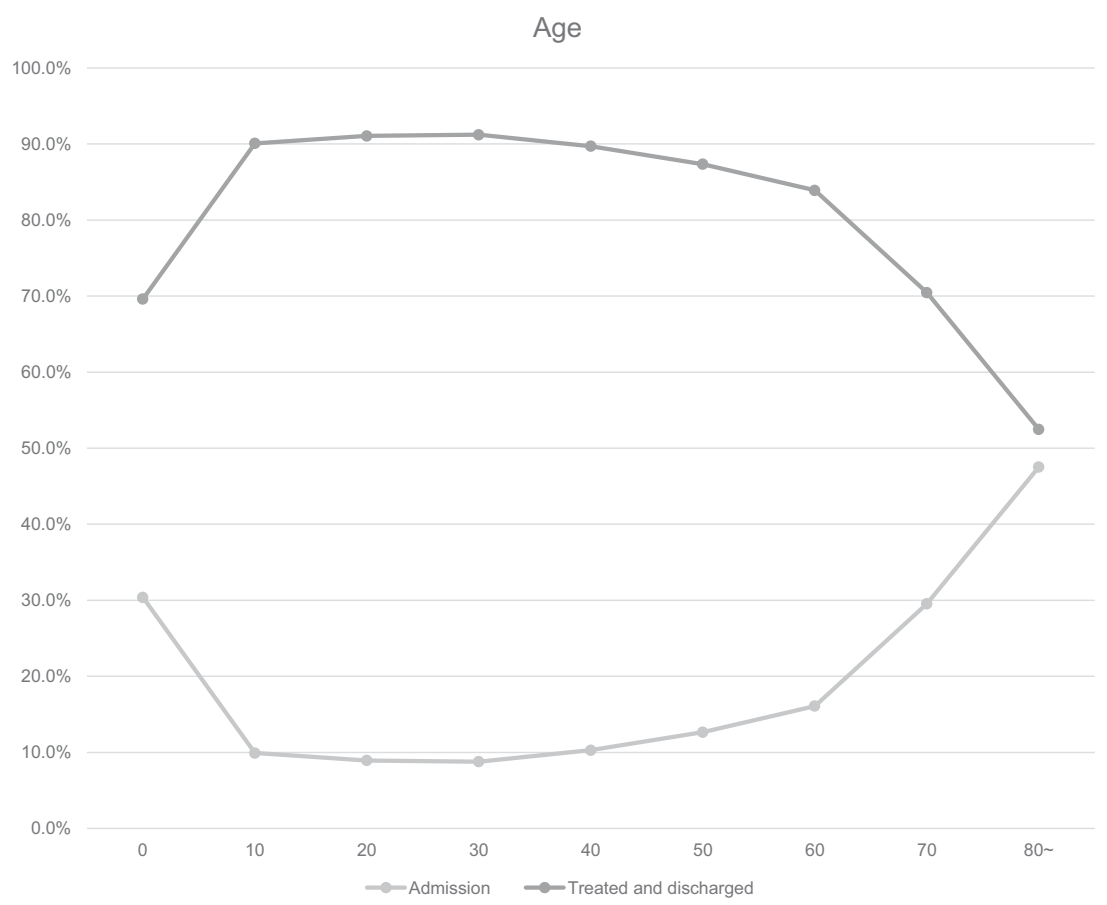

Figure 4. Ratio of Emergency Department visits by age. Ratio of total Emergency Department visits, admission, treated and discharged patients for urolithiasis in Korea from 2014 to 2016 in the NEDIS stratified by age.

Since NEDIS data includes data from national ED, they are important in terms of analysis of the characteristics of patients visiting Korean ED. The government operated NEDIS includes the clinical and administrative data of all patients visiting $\mathrm{ED}^{11}$. The data collected include age, sex, type of insurance, an initial vital signs, the visit flow, date of visit and discharge.

Acute treatment for urolithiasis is mainly aimed at controlling symptoms. Therefore, in cases in which subjective indicators are treated, different factors may influence treatment outcomes and these may subjected to social influences. Thus, medical costs, the type of insurance coverage, and treating hospital, social environment of the hospital visit may influence the patient through different mechanisms and may ultimately lead to a pattern of hospitalization $^{12}$.

This study showed that the number of urolithiasis patients visiting the EDs increased gradually, however the proportion of urolithiasis patients among all ED patients remained constant. Similarly, Roghmann et al. reported that the number of ED patients remained stable ${ }^{13}$. Other national studies have reported that the proportion of 
urolithiasis patients among ED patients was $1.8 \%$, but these were based on a single year; thus, any increase in was impossible to determine ${ }^{14}$. With regard to the prevalence of urinal stones, some studies have reported a gradual increase, with lifetime cumulative incidence reaching up to $37 \%^{4,9,15,16}$. Currently, it has been reported that urolithiasis was increasing in Asia due to the influence of westernized culture ${ }^{4,17}$. Taylor et al. showed that obesity and metabolic syndrome could influence the occurrence of urolithiasis ${ }^{10}$. Thus, ED visits of urolithiasis patients may differ in terms of country, society, and period; therefore, conducting additional epidemiological research is important.

In this study, the hospitalization rate of urolithiasis patients was approximately $13.2 \%$. Urolithiasis occurred more frequently in men, despite more female patients being hospitalized. In addition, the hospitalization rate of patients aged $<9$ years was $30.4 \%$, and those aged $\geq 70$ years had a higher hospitalization rate. The annual rate of hospitalized patients was not significantly different. Ghani et al. ${ }^{18}$ reported that the hospitalization rate of upper urinary tract stone patients in the United States was $12 \%$, a result that was similar to that of this study. Moreover, the increased trend in hospitalization rate observed in the American study was not significant, which was similar to this study. Although the reasons for which the hospitalization rate remained constant may vary, it may be due to more accurate diagnosis based on non-contrast CT and better pain control of spontaneous stone passage during medical treatment ${ }^{19-21}$. With the rapid universalization of CT images, clinicians are able to receive information on the existence of stones and on their location and size. Such information is considered to be helpful to determine drug treatment and hospitalization.

Korea has four different seasons: spring (March to May), summer (June to August), fall (September to November), and winter (December to February.). Numerous reports have confirmed the seasonal variation of urolithiasis. It is well known that when temperature increases, the discharge of calcium through urine increases, or calcium oxalate or calcium phosphate excessively saturates, and thus the potential for urolithiasis formation increases $^{22-24}$. Furthermore, the frequency of urolithiasis occurrence was higher in the months of August, July, and May in order. Nevertheless, months with higher frequency of occurrence did not correspondingly have higher hospitalization rates. Lin et al. ${ }^{25}$, in a study from Taiwan, investigated the relationship between ESWL, the main treatment for urolithiasis, and environmental temperature. The ESWL count showed a strong association with temperature. They also suggested that excessive sweating in hot weather led to a reduction in urinary output and concentration, which resulted in increased stone formation. Sirohi et al. ${ }^{26}$ revealed that there was a close relationship with temperature based on an analysis of the changes in monthly temperature in New York.

According to Hong et al. ${ }^{14}$, who surveyed patients aged $\geq 18$ years in selected EDs in 2010, a year earlier than the start year of the data used in this study, the average age of the subjects was 45 years, and the male-to-female ratio was 2:1. Most study subjects visited the EDs between 6-10 a.m. and the highest number of visits occurred in August. The main symptoms reported by the study subjects were flank pain, abdominal pain, and hematuria in order. The average stay in the ED was 171 hours and women remained longer than men. Park et al. ${ }^{27}$ also revealed that women visited EDs twice as often than men, just as in this study. Most studies have shown that the prevalence of urolithiasis was higher in men and had a hospitalization rate of approximately $6 \%-8 \%$, which differed from the results of this study (13.2\%), a difference likely attributable to the lack of young children $<18$ years included in the study. Indeed, the hospitalization rate of children aged $<9$ years among ED patients was high (30.4\%). In this study, the stay in the ED was $2.6 \pm 11.3$ hours on average and for hospitalized patients this stay was longer, which was likely influenced by patients whose main indication for hospitalization of urolithiasis was uncontrolled pain. The indication for hospitalization occurs when a patient's pain worsens in the ED despite different attempts at its alleviation. Such patients will remain in the ED longer than patients whose pain improves and return home after treatment. Bae et al. ${ }^{28}$ reported that upper urinary stones were found most often among patients in their $40 \mathrm{~s}$. In our study, the number of ED patients in their 40s was the highest. In addition, according to this study, most visits occurred at 7 a.m., and a further peak was observed at 9 p.m. (Fig. 1). However, the hospitalization rate of ED patients following daytime visits was higher. A larger number of ED visits occurred in the hotter season, at 7 a.m., in men, on weekends, and by those in their 50s; however, the hospitalization rate of such groups was not higher than that of other groups. Instead, a higher hospitalization rate occurred in November, and comprised women, on weekday visits, and for patients $<9$ years old and for those aged $\geq 70$ years.

This study has some limitations. First, patients with a diagnosis defined as simple abdomen were not subjected to additional examination and were likely to be excluded. Patient data were extracted basis on the diagnostic definition given. For this reason, patients whose symptoms only were descriptive may not have received a definitive diagnosis. Nevertheless, patients who were definitely diagnosed with urolithiasis through additional examinations, rather than clinical diagnosis alone, were more likely to have received a specific diagnosis definition in the database. Therefore, few urolithiasis patients appeared to have been omitted. Secondly, despite the database-based extraction, there were few clinical data registered regarding diagnostic modalities, individual treatments or prognosis. Therefore, further clinical research will be necessary. Finally, the authors did not look into factors that lead to hospitalization, the results should be interpreted with caution.

\section{Conclusion}

This is the first study to analyze the characteristics of urolithiasis patients visiting ED based on a national database comprising patients of all age groups. Urolithiasis patients accounted for $1.3 \%$ of all patients who visited ED and of these, those requiring hospitalization for treatment accounted for $13.2 \%$. Female patients had a higher hospitalization rate than males. Of patients aged $<9$ years and those aged $\geq 70$ years older, those with medical insurance had a higher hospitalization rate. More visits occurred in hot seasons, on weekends, and in the 6 a.m. and 8 p.m. slots. The characteristics of urolithiasis patients who visited an ED described by this study will help shape the treatment approach for these patients in an ED and will help guide the management of ED resources. It is necessary to continue collecting basic patient data from those who visit domestic medical centers. 
Received: 8 April 2019; Accepted: 17 October 2019;

Published online: 12 November 2019

\section{References}

1. Graham, A., Luber, S. \& Wolfson, A. B. Urolithiasis in the emergency department. Emerg. Med. Clin. North Am. 29, 519-538 (2011).

2. Tae, B. S., Balpukov, U., Cho, S. Y. \& Jeong, C. W. Eleven-year Cumulative Incidence and Estimated Lifetime Prevalence of Urolithiasis in Korea: a National Health Insurance Service-National Sample Cohort Based Study. J. Korean Med. Sci. 33, e13 (2018).

3. Teichman, J. M. Clinical practice. Acute renal colic from ureteral calculus. N. Engl. J. Med. 350, 684-693 (2004).

4. Jeong, I. G. et al. Association between metabolic syndrome and the presence of kidney stones in a screened population. Am. J. Kidney Dis. 58, 383-388 (2011).

5. Hesse, A., Brandle, E., Wilbert, D., Köhrmann, K. U. \& Alken, P. Study on the prevalence and incidence of urolithiasis in Germany comparing the years 1979 vs. 2000. European urology 44, 709-713 (2003).

6. Wilkinson, H. Clinical investigation and management of patients with renal stones. Annals of clinical biochemistry 38, 180-187 (2001).

7. Bultitude, M. \& Rees, J. Management of renal colic. BMJ 345, e5499 (2012).

8. Leveridge, M. et al. Renal colic: current protocols for emergency presentations. Eur. J. Emerg. Med. 23, 2-7 (2016).

9. Pearle, M. S., Calhoun, E. A. \& Curhan, G. C. \& Urologic Diseases of America Project. Urologic diseases in America project: urolithiasis. The Journal of urology 173, 848-857 (2005).

10. Taylor, E. N., Stampfer, M. J. \& Curhan, G. C. Obesity, weight gain, and the risk of kidney stones. Jama 293, 455-462 (2005).

11. Cha, W. C., Ahn, K. O., Shin, S. D., Park, J. H. \& Cho, J. S. Emergency Department Crowding Disparity: a Nationwide CrossSectional Study. J. Korean Med. Sci. 31, 1331-1336 (2016).

12. Eaton, S. H. et al. Admission rates and costs associated with emergency presentation of urolithiasis: analysis of the Nationwide Emergency Department Sample 2006-2009. Journal of endourology 27, 1535-1538 (2013).

13. Roghmann, F. et al. Incidence and treatment patterns in males presenting with lower urinary tract symptoms to the emergency department in the United States. J. Urol. 190, 1798-1804 (2013).

14. Hong, D. Y., Kim, J. W., Lee, K. R., Park, S. O. \& Baek, K. J. Epidemiologic and Clinical Characteristics of Patients Presenting with Renal Colic in Korea. Urology journal 12, 2148-2153 (2015).

15. Scales, C. D. Jr., Smith, A. C., Hanley, J. M. \& Saigal, C. S. \& Urologic Diseases in America Project. Prevalence of kidney stones in the United States. European urology 62, 160-165 (2012).

16. Stamatelou, K. K., Francis, M. E., Jones, C. A., Nyberg, L. M. \& Curhan, G. C. Time trends in reported prevalence of kidney stones in the United States: 1976-1994. Kidney international 63, 1817-1823, (2003).

17. Kim, Y. J. et al. Changes in urinary lithogenic features over time in patients with urolithiasis. Urology 74, 51-55 (2009).

18. Ghani, K. R. et al. Emergency department visits in the United States for upper urinary tract stones: trends in hospitalization and charges. J. Urol. 191, 90-96 (2014).

19. Westphalen, A. C., Hsia, R. Y., Maselli, J. H., Wang, R. \& Gonzales, R. Radiological imaging of patients with suspected urinary tract stones: national trends, diagnoses, and predictors. Academic emergency medicine: official journal of the Society for Academic Emergency Medicine 18, 699-707 (2011)

20. Brede, C., Hollingsworth, J. M., Faerber, G. J., Taylor, J. S. \& Wolf, J. S. Jr. Medical expulsive therapy for ureteral calculi in the real world: targeted education increases use and improves patient outcome. The Journal of urology 183, 585-589 (2010).

21. Phillips, E., Kieley, S., Johnson, E. B. \& Monga, M. Emergency room management of ureteral calculi: current practices. Journal of endourology 23, 1021-1024 (2009).

22. Chi, B. H. et al. Daily Mean Temperature and Urolithiasis Presentation in Six Cities in Korea: Time-Series Analysis. J. Korean Med. Sci. 32, 999-1008 (2017).

23. Park, H. K. et al. The effect of climate variability on urinary stone attacks: increased incidence associated with temperature over 18 degrees C: a population-based study. Urolithiasis 43, 89-94 (2015).

24. Eisner, B. H. et al. The effects of ambient temperature, humidity and season of year on urine composition in patients with nephrolithiasis. BJU international 110, E1014-1017 (2012).

25. Lin, K. J. et al. The impact of climate factors on the prevalence of urolithiasis in Northern Taiwan. Biomed J 37, 24-30 (2014).

26. Sirohi, M., Katz, B. F., Moreira, D. M. \& Dinlenc, C. Monthly variations in urolithiasis presentations and their association with meteorologic factors in New York City. J. Endourol. 28, 599-604 (2014).

27. Park, J., Suh, B., Lee, M. S., Woo, S. H. \& Shin, D. W. National Practice Pattern and Time Trends in Treatment of Upper Urinary Tract Calculi in Korea: a Nationwide Population-Based Study. J. Korean Med. Sci. 31, 1989-1995 (2016)

28. Bae, S. R. et al. The epidemiology of reno-ureteral stone disease in Koreans: a nationwide population-based study. Urolithiasis 42 , 109-114 (2014).

\section{Acknowledgements}

This research used National Emergency Department Information System (NEDIS) data (N20180520611) provided by the NEDIS. This research was supported by Korea University Grant (K1924991).

\section{Author contributions}

K.J.W., K.J.Y. and P.H.S. conceived the study. K.J.W. and K.J.Y. collected the data, performed the statistical analysis K.J.W. and K.J.Y. wrote the main manuscript text. A.S.T., O.M.M., M.D.G. helped to conceive the study and draft the manuscript. All authors reviewed the manuscript.

\section{Competing interests}

The authors declare no competing interests.

\section{Additional information}

Correspondence and requests for materials should be addressed to H.S.P.

Reprints and permissions information is available at www.nature.com/reprints.

Publisher's note Springer Nature remains neutral with regard to jurisdictional claims in published maps and institutional affiliations. 
(c) (i) Open Access This article is licensed under a Creative Commons Attribution 4.0 International License, which permits use, sharing, adaptation, distribution and reproduction in any medium or format, as long as you give appropriate credit to the original author(s) and the source, provide a link to the Creative Commons license, and indicate if changes were made. The images or other third party material in this article are included in the article's Creative Commons license, unless indicated otherwise in a credit line to the material. If material is not included in the article's Creative Commons license and your intended use is not permitted by statutory regulation or exceeds the permitted use, you will need to obtain permission directly from the copyright holder. To view a copy of this license, visit http://creativecommons.org/licenses/by/4.0/.

(C) The Author(s) 2019 